\title{
On Some Geometry and Equivalence Classes of Normal Form Games
}

\author{
Fabrizio Germano* \\ Departament d'Economia i Empresa \\ Universitat Pompeu Fabra \\ Ramon Trias Fargas 25-27 \\ 08005 Barcelona, Spain \\ fabrizio.germano@econ.upf.es
}

March 2003

\begin{abstract}
Equivalence classes of normal form games are defined using the geometry of correspondences of standard equilibrium concepts like correlated, Nash, and robust equilibrium, or risk dominance and rationalizability. Resulting equivalence classes are fully characterized and compared across different equilibrium concepts for $2 \times 2$ games. It is argued that the procedure can lead to broad and gametheoretically meaningful distinctions of games as well as to alternative ways of viewing and testing equilibrium concepts. Larger games are also briefly considered. Keywords Non-cooperative games, classification and equivalence classes, experimental games, geometry of games. JEL Classification C70, C72, C78, C90, C91.
\end{abstract}

\footnotetext{
*I thank Vince Crawford, Itzhak Gilboa, Jean-François Mertens, Rosemarie Nagel, Jack Robles, and Joel Sobel for insightful comments and conversations. Financial support from European Commission, TMR Network Grant ERBFMRXCT0055, "Cooperation and Information," as well as in the form of a Ramon y Cajal fellowship from the Spanish Ministry of Science and Technology are gratefully acknowledged. All errors are my own.
} 


\section{Introduction}

The representation of a strategic situation by means of a normal form game is one of the most fundamental constructions of game theory. But, while much work has been done devising and studying a variety of equilibrium concepts for both normal and extensive form games, not much has been done to systematically differentiate the infinite number of games that naturally arise in theory and practice. In other words, questions like, What types of games are there? or, What are meaningful distinctions between different strategic situations? are hardly addressed in a systematic way in the game theory literature. (In Section 5, we discuss some exceptions and other related literature.)

The present paper attempts to address such questions by means of a general geometric procedure involving standard (non-cooperative) equilibrium concepts such as rationalizability, correlated, or Nash equilibrium. The idea is that the geometry of a given equilibrium correspondence reflects basic aspects of the logic or rationality underlying the equilibrium concept and can therefore be used to differentiate or identify games.

Using the geometry of equilibrium correspondences allows to identify games that from other points of view would be regarded as different. Consider the game

$$
\gamma_{\epsilon}=\left(\begin{array}{ll}
(1,0) & (0,1) \\
(0,1) & (1, \epsilon)
\end{array}\right) .
$$

For $\epsilon=0$ this is a constant-sum game, while for any other value of $\epsilon$ it does not qualify as such. Yet, it seems clear that for values of $\epsilon$ close to zero, the game retains the "character" of a constant-sum or at least of a matching pennies type game. Part of our task is how to formally define notions of equivalence or of "types" of games. As we will see, counting numbers of, e.g., Nash equilibria or rationalizable strategies, as a way of distinguishing games, is not unrelated to looking at some geometric features of the two correspondence; however, it gives distinct classes as we will see.

More specifically, the geometric features we consider are simply the discontinuities of given (equilibrium) correspondences, in the following sense. Take a correspondence $e: \mathbb{R}^{m} \rightarrow X$, where $\mathbb{R}^{m}$ is a space of games, e.g., a space of payoff parameters, and where $X$ is a set, e.g., the space of mixed strategies. The discontinuities of $e$, or more precisely, the games at which $e$ is discontinuous, divide the space of games $\mathbb{R}^{m}$ into a certain number of connected components. All games within the same connected component are then said to be equivalent relative to the correspondence $e$. For example, the Nash equilibrium correspondence is continuous in a neighborhood around the above game $\gamma_{\epsilon}$ with $\epsilon=0$, implying that all games in such a 
neighborhood are what we call Nash equivalent. Moreover, two games that differ only by relabeling the strategies and/or the players, are also said to be equivalent, so that two games in two disconnected components may also be equivalent. Given that for semi-algebraic correspondences, which include the Nash, correlated, and rationalizable correspondences among others, the number of resulting connected components is always finite, (see, e.g., Schanuel et al. (1991) and Blume and Zame (1994)), and since the number of connected components is an upper bound for the number of equivalence classes distinguished, this already implies that the classifications obtained for semi-algebraic correspondences distinguish finitely many equivalence classes of games.

Hence, besides giving a sense of which aspects of normal form games different equilibrium concepts distinguish and which aspects they ignore, the classifications also provide (natural) organizations of the underlying spaces of games into well-defined and typically finite equivalence classes. Not only does this provide a better understanding of the underlying equilibrium concepts, and especially of the geometries of the equilibrium correspondences, but it also provides a particularly transparent way of comparing equilibrium concepts with each other. At the same time, the present approach forces one to think about what the fundamental characteristics describing a strategic situation may be and why.

After formally defining the procedure, we consider the space of $2 \times 2$ games and show that reasonable distinctions are obtained in this case. In particular, we show that, applying the procedure to the rationalizability, correlated, and Nash equilibrium concepts, leads to the same three classes of generic $2 \times 2$ games being distinguished, namely, (a-i) games of the matching pennies type (or with zero pure Nash equilibria), (a-ii) games solvable by iterated strict dominance (or with unique pure Nash equilibrium), and (a-iii) coordination type games (or with two pure Nash equilibria). While the distinction of the three classes already has been made, (see, e.g., Rapoport and Guyer (1966)), it is not immediately obvious why, e.g., games in (a-i) and (a-ii) are not equivalent relative to rationalizability, since in both cases all strategies are rationalizable; similarly, games in (a-i) and (a-ii) are not equivalent relative to Nash or correlated equilibrium, despite all games in both classes always having a unique Nash and a unique correlated equilibrium. However, given that such a classification of $2 \times 2$ games has already been obtained (even if by other means), a more basic contribution of this paper is the procedure itself, which applies to arbitrary finite normal form games and which generally yields different classifications, depending on the equilibrium concept used.

In fact, we further show that the same procedure, applied to the concept 
of robust equilibrium of Kajii and Morris (1997a, b), yields, again within generic $2 \times 2$ games, a different classification than the one above, ${ }^{1}$ namely, (b-i) games of the matching pennies type on one hand, and (b-ii) games solvable by iterated strict dominance and coordination games on the other. We regard the fact the two classifications are not the same and, in particular, that the two types of games under (b-ii) are equivalent with respect to the robust equilibrium concept, as an important difference. Essentially, it is related to the fact that games of the form

$$
\hat{\gamma}_{\epsilon}=\left(\begin{array}{ll}
(1,1) & (0,0) \\
(0,0) & (\epsilon, \epsilon)
\end{array}\right)
$$

are all equivalent relative to the robust equilibrium concept, regardless of whether $\epsilon$ is positive or negative, as long as it is not equal to $1 .{ }^{2}$ This is not true for the other three concepts, since the set of rationalizable strategies as well as the sets of correlated and Nash equilibria all change abruptly as $\epsilon$ goes from being negative to being positive. We briefly argue in the paper, that experimental evidence seems to be more supportive of the classification obtained with robust equilibria, at least in the $2 \times 2$ case. However, we believe that experiments, testing equilibrium concepts relative to the equivalence classes implied, have only partially been performed, and think that, in principle, they could provide useful tests of equilibrium theories.

The paper is organized as follows. Section 2 introduces preliminary notation and definitions, Section 3 defines and analyzes equivalence classes relative to the notions of rationalizability, Nash, and correlated equilibrium, with an emphasis on $2 \times 2$ games. Section 4 considers robust and risk dominant equilibria. Finally, Section 5 discusses related literature, and Section 6 concludes.

\section{Preliminary Notions}

Let $I=\{1, \ldots, n\}$ denote the set of players, let $S_{i}$ denote player $i$ 's space of pure strategies, and $S=\times_{i \in I} S_{i}$ the space of pure strategy profiles. Let $\Sigma_{i}=\Delta\left(S_{i}\right)$ denote the set of probability distributions on $S_{i}, \Sigma=\times_{i \in I} \Sigma_{i}$ the space of mixed strategies, and $\Delta(S)$ the probability measures on $S$. Let also $S_{-i}=\times_{j \neq i} S_{j}$, and $\Sigma_{-i}=\times_{j \neq i} \Sigma_{j}$ and set $K_{i}=\# S_{i}, K=\sum_{i \in I} K_{i}$,

\footnotetext{
${ }^{1}$ For generic $2 \times 2$ games, the robust equilibrium is unique and coincides with the unique risk dominant equilibrium in the sense of Harsanyi and Selten (1988); see Kajii and Morris (1997a, b).

${ }^{2}$ These games have a unique robust equilibrium, which is top-left if $\epsilon<1$ and bottomright if $\epsilon>1$; relabeling the strategies makes them all equivalent relative to robust (or risk dominant) equilibrium.
} 
$\kappa=\prod_{i \in I} K_{i}$. In what follows, we consider finite normal form games, i.e., where $n$ and each $K_{i}$ are finite, and fix both the set of players and the set of strategy profiles, so that we can identify a game with a point in Euclidean space $\gamma \in \mathbb{R}^{\kappa n}$ and, accordingly, the space of games with the whole Euclidean space $\mathbb{R}^{\kappa n}$. We also denote by $\gamma^{i} \in \mathbb{R}^{\kappa}$ the payoff array of player $i$.

For a given normal form game, $\gamma \in \mathbb{R}^{\kappa n}$, denote by $e_{R A T}(\gamma) \subset S$ the set of rationalizable strategy profiles, by $e_{N E}(\gamma) \subset \Sigma$ the set of Nash equilibria, and by $e_{C E}(\gamma) \subset \Delta(S)$ the set of correlated equilibria. ${ }^{3}$ Further, denote by $e_{R A T}, e_{N E}$, and $e_{C E}$ the respective correspondences mapping games to corresponding sets of equilibria, and by $\eta_{R A T} \subset \mathbb{R}^{k^{n}} \times S$, $\eta_{N E} \subset \mathbb{R}^{k n} \times \Sigma$, and $\eta_{C E} \subset \mathbb{R}^{\kappa n} \times \Delta(S)$ the respective graphs of the equilibrium correspondences.

The definition of equivalence class that is given below, builds on two ingredients; the first is based on continuity of equilibrium or related correspondences, and the second is based on the fact that relabeling players and their strategies does not really change the game. The following lemma follows from the fact that $e_{R A T}, e_{C E}$, and $e_{N E}$ are semi-algebraic. ${ }^{4}$

Lemma 1 There exist closed, lower dimensional subsets of the space of games, $D_{R A T}, D_{C E}$, and $D_{N E} \subset \mathbb{R}^{\kappa n}$, such that $e_{R A T}, e_{C E}$, and $e_{N E}$ are continuous respectively on the complements $\mathbb{R}^{\kappa n} \backslash D_{R A T}, \mathbb{R}^{\kappa n} \backslash D_{C E}$, and $\mathbb{I R}^{\kappa n} \backslash D_{N E}$. Moreover, each of the complements consists of a finite number of connected components.

The nature of the complements $\mathbb{R}^{\kappa n} \backslash D_{R A T}$ and $\mathbb{R}^{\kappa n} \backslash D_{N E}$ will play an important role in the classifications. Notice that, since rationalizabity and interated strict dominance are equivalent for two-player games, (see, e.g., Fudenberg and Tirole (1991), Ch. 2, p. 51), it follows that, at any game $\gamma \in \mathbb{R}^{2 \kappa} \backslash D_{R A T}$, the number of strategies surviving iterated strict dominance is locally constant. Also, since, for two-player games, continuity of $e_{N E}$ is equivalent to all Nash equilibria being locally unique, (see van Damme (1991), Ch. 3, p. 55, where strongly stable corresponds to what we call locally unique), implies that, at any game $\mathbb{R}^{\kappa n} \backslash D_{N E}$, the set of Nash equilibria consists of a finite (and odd) number of locally unique Nash equilibria. 5

\footnotetext{
${ }^{3}$ See Fudenberg and Tirole (1991) or Myerson (1991) for definitions.

${ }^{4}$ Schanuel et al. (1991) prove the case $e_{N E}$; the cases $e_{R A T}$ and $e_{C E}$ (as well as of some of the other correspondences defined in this paper) are analogous; see Lemma 5 in the appendix for more details.

${ }^{5}$ Let $(\gamma, x) \in \eta$ be a point on the graph of the (equilibrium) correspondence $e$ : $\mathbb{R}^{k n} \rightarrow X$, we say the equilibrium $x \in X$ of $\gamma \in \mathbb{R}^{k n}$ is locally unique if there exists
} 
The other important ingredient in the definition of equivalence class, can be formalized by means of certain (linear) maps, which, following Nash (1951), we call symmetry operations. These maps identify games through relabeling of the players' strategies and/or of the players themselves. ${ }^{6}$ More precisely, let $P_{a}$ be the set of all permutations $p_{a}: I \rightarrow I$ satisfying $K_{p_{a}(i)}=$ $K_{i}, i \in I$, let $P_{s}$ be the set of all permutations $p_{s}=\left(p_{s}^{i}: S_{i} \rightarrow S_{i}\right)_{i \in I}$, and let:

$$
\begin{aligned}
& \Psi_{a}=\left\{\psi_{a}: \mathbb{R}^{\kappa n} \rightarrow \mathbb{R}^{\kappa n} \mid \psi_{a}(\gamma)=\left(\gamma_{k_{p_{a}^{-1}(1)}^{p_{a}(i)}, \ldots, k_{p_{a}^{-1}(n)}}\right)_{i \in I}, p_{a} \in P_{a}, \gamma \in \mathbb{R}^{\kappa n}\right\}, \\
& \Psi_{s}=\left\{\psi_{s}: \mathbb{R}^{\kappa n} \rightarrow \mathbb{R}^{\kappa n} \mid \psi_{s}(\gamma)=\left(\gamma_{p_{s}^{1}\left(k_{1}\right), \ldots, p_{s}^{n}\left(k_{n}\right)}^{i}\right)_{i \in I}, p_{s} \in P_{s}, \gamma \in \mathbb{R}^{\kappa n}\right\} .
\end{aligned}
$$

We say a map $\psi: \mathbb{R}^{\kappa n} \rightarrow \mathbb{R}^{\kappa n}$ is a symmetry operation within the class of $n$-person $K_{1} \times \cdots \times K_{n}$ games, if $\psi=\psi_{a} \psi_{s}$ for some $\psi_{a} \in \Psi_{a}$, $\psi_{s} \in \Psi_{s}$, and denote by $\Psi$ the set of all such symmetry operations. This space depends on the number of players and the cardinality of the strategy spaces, i.e., on $K_{1}, \ldots, K_{n}$, which, to save notation, we often leave out. The maps $p_{a} \in P_{a}, p_{s} \in P_{s}$ correspond respectively to relabeling of the players and of the players' strategies. The maps $\psi_{a} \in \Psi_{a}, \psi_{s} \in \Psi_{s}$ are the maps induced on the space of games by corresponding maps $p_{a}$ and $p_{s}$. Notice that elements of $\Psi$ are matrices with determinant + or -1 . Next, we introduce the notion of equivalence.

Definition 1 Let $\mathbb{R}^{\kappa n}$ be the space of $K_{1} \times \cdots \times K_{n}$ games in normal form with $n$ players and let $e: \mathbb{R}^{\kappa n} \rightarrow X$ be an equilibrium correspondence on this space of games, we say two games $\gamma_{0}, \gamma_{1} \in \mathbb{R}^{\kappa n}$ are equivalent relative to $e$, which we denote by $\gamma_{0} \sim \gamma_{1}$, if there exists a symmetry operation $\psi \in \Psi\left(K_{1}, \ldots, K_{n}\right)$, and a continuous path $v:[0,1] \rightarrow \mathbb{R}^{\kappa n}$, with $v(0)=\gamma_{0}$ and $v(1)=\psi\left(\gamma_{1}\right)$, such that, for all $\gamma \in v([0,1])$, e is continuous at $\gamma$.

Since we require that the correspondence $e$ be continuous at all games along the path $v$ - including the games at the endpoints $\gamma_{0}$ and $\psi\left(\gamma_{1}\right)-$ we in fact define equivalence only for a subset of $\mathbb{R}^{\kappa n}$. As mentioned, if the correspondence $e$ is semi-algebraic, then the subset is open and dense in $\mathbb{R}^{\kappa n}$ and the set of games at which the correspondence is discontnuous will be contained in a lower dimensional subset (see Lemmas 1 and 5). The following shows that Def. 1 indeed defines an equivalence relation.

Lemma 2 The relation $\sim$ defines an equivalence relation on the space of games in $\mathbb{R}^{\kappa n}$ at which e is continuous.

a neighborhood $U$ of $\gamma$ and a neighborhood $V$ of $x$ such that (i) $\left|e\left(\gamma^{\prime}\right) \cap V\right|=1$, for all $\gamma^{\prime} \in U$, and (ii) the map $h: U \rightarrow V, h\left(\gamma^{\prime}\right)=e\left(\gamma^{\prime}\right) \cap V$ is continuous. Locally unique equilibria can be parameterized (locally) by games.

${ }^{6}$ For a thorough treatment of such symmetries within a more abstract space of games, the reader is referred to Mertens (1987) and the notions of ordinality defined there. 


\section{Some Standard Equivalence Classes}

Together with Def. 1 , we say that two games $\gamma_{0}, \gamma_{1} \in \mathbb{R}^{\kappa n}$ are respectively rationalizable and Nash equivalent, which we denote respectively by $\gamma_{0} \sim_{R A T} \gamma_{1}$ and $\gamma_{0} \sim_{N E} \gamma_{1}$, if they are equivalent relative to respectively $e_{R A T}$ and $e_{N E}$. While we could define the equivalence classes analogously for correlated equilibria, to simplify the analysis, we say that two games $\gamma_{0}, \gamma_{1} \in \mathbb{R}^{\kappa n}$ are correlated equivalent, which we denote by $\gamma_{0} \sim_{C E} \gamma_{1}$, if they are equivalent relative to the correspondence

$$
e_{d C E}: \mathbb{R}^{\kappa n} \rightarrow \mathbb{R}, e_{d C E}(\gamma)=\operatorname{dim}\left[e_{C E}(\gamma)\right]
$$

which assigns to any game $\gamma$, the dimension of the corresponding set of correlated equilibria. Since the the set of correlated equilibria of a given game is always a nonempty, compact, and convex polyhedron, defined by a finite number of linear inequalities, its dimension is always well-defined. It turns out that checking for changes in the dimension of the set of equilibria (continuity of $e_{d C E}$ ) is much easier than checking for continuity of $e_{C E}$. Although in principle, changes in the dimension could occur at points where $e_{C E}$ is continuous, we view the changes in dimension themselves as significant geometric or topological events of the correspondence $e_{C E}$.

\section{1 $2 \times 2$ Games}

In this section we consider the case where $n=2$ and also $K_{1}=K_{2}=2$. We will see how the equivalence classes defined above make game-theoretically meaningful distinctions, and lead to the same characterizations in this case. However, before giving the full characterizations of the rationalizable, correlated and Nash equivalence classes for this case, we first introduce the notion of a nondegenerate two-player game, (see von Stengel (2002)), which we use to compute and describe the standard equivalence classes.

Definition 2 Let $\gamma \in \mathbb{R}^{2 \kappa}$ be a two-player game in normal form. We say the game $\gamma$ is nondegenerate if any mixed strategy $x \in \Sigma_{i}$ of player $i$ has at most $|\operatorname{supp}(x)|$ pure best responses, for $i=1,2$; we also say $\gamma$ is degenerate if it is not nondegenerate.

Accordingly, we say that two games $\gamma_{0}, \gamma_{1} \in \mathbb{R}^{2 \kappa}$ are nondegenerate equivalent, which we denote by $\gamma_{0} \sim_{N D} \gamma_{1}$, if they are equivalent relative to the correspondence

$$
e_{N D}: \mathbb{R}^{2 \kappa} \rightarrow[0,1], e_{N D}(\gamma)= \begin{cases}1 & \text { if } \gamma \text { is degenerate } \\ 0 & \text { else }\end{cases}
$$


Furthermore, for any nondegenerate two-player game $\gamma \in \mathbb{R}^{2 \kappa}$, let the set $[\gamma]=\left\{\gamma^{\prime} \in \mathbb{R}^{2 \kappa} \mid \gamma^{\prime} \sim_{N D} \gamma\right\}$ denote the nondegenerate equivalence class associated to $\gamma$; let also $\left[\gamma, \gamma^{\prime}\right]=\left([\gamma] \cup\left[\gamma^{\prime}\right] \cup\left(\operatorname{cl}[\gamma] \cap \operatorname{cl}\left[\gamma^{\prime}\right]\right)\right)^{\circ}$, for $\gamma, \gamma^{\prime} \in \mathbb{R}^{2 \kappa}$ nondegenerate with $[\gamma] \cap\left[\gamma^{\prime}\right]=\emptyset$, and where $\operatorname{cl}(\cdot)$ denotes the closure and $(\cdot)^{\circ}$ denotes the interior. ${ }^{7}$

The importance of the nondegenerate games and nondegenerate equivalence classes for the equivalence classes resulting from the standard equilibrium concepts mentioned above, follows from the following result.

Proposition 1 If two two-player games are nondegenerate equivalent, then they are also rationalizable, correlated, and Nash equivalent, and there are no pure stategies of any player that are weakly dominated by or payoff equivalent to a mixed strategy of the same player that is not itself strictly dominated.

This shows that the rationalizable, correlated, and Nash equivalence classes have the more basic nondegenerate equivalence classes in common, or, in other words, their intersections contain the nondegenerate equivalence classes. We view this as evidence that the players' best-reply correspondences are in a specific sense fundamental to the three equilibrium notions.

We are finally in a position to compute the standard equivalence classes for the $2 \times 2$ two-player games. It is easy to check that, in this case, the singular games are games with the property $\gamma_{1}^{i}=\gamma_{2}^{i}$ for $i=1$ or $i=2$ (or both). Moreover, the singular $2 \times 2$ games divide the space of all $2 \times 2$ games, (which we identify with $\mathbb{R}^{8}$ ), into 16 connected components of nondegenerate games. (Notice that by Prop. 1 all games within any one of the 16 connected components are rationalizable, correlated, and Nash equivalent.) Applying the symmetry operations of relabeling the players' strategies, further identifies some of the 16 components and leads to 5 classes of nondegenerate games, which can be unambiguously represented by the following games:

$$
\begin{gathered}
\gamma_{1}=\left(\begin{array}{cc}
(1,1) & (1,0) \\
(0,1) & (0,0)
\end{array}\right), \gamma_{2}=\left(\begin{array}{ll}
(1,1) & (1,0) \\
(0,0) & (0,1)
\end{array}\right), \gamma_{3}=\left(\begin{array}{ll}
(1,1) & (0,0) \\
(0,1) & (1,0)
\end{array}\right) \\
\gamma_{4}=\left(\begin{array}{ll}
(1,0) & (0,1) \\
(0,1) & (1,0)
\end{array}\right), \gamma_{5}=\left(\begin{array}{ll}
(1,1) & (0,0) \\
(0,0) & (1,1)
\end{array}\right) .
\end{gathered}
$$

Applying the symmetry operation of relabeling the players, allows to further identify $\gamma_{2}$ and $\gamma_{3}$, (i.e., $\gamma_{2} \sim_{N D} \gamma_{3}$ ), which means that there are exactly

\footnotetext{
${ }^{7}$ In the definition of $\left[\gamma, \gamma^{\prime}\right]$, besides the nondegenerate games in $[\gamma] \cup\left[\gamma^{\prime}\right]$, we also include all degenerate games that lie between two neighboring connected components of $[\gamma]$ and $\left[\gamma^{\prime}\right]$, which are precisely the games in $\mathrm{cl}[\gamma] \cap \mathrm{cl}\left[\gamma^{\prime}\right]$ without points on the boundary.
} 
four nondegenerate equivalence classes: $\left[\gamma_{1}\right],\left[\gamma_{2}\right],\left[\gamma_{4}\right]$, and $\left[\gamma_{5}\right]$. We state this formally.

Lemma 3 For the $2 \times 2$ two-player games, there are exactly four distinct nondegenerate equivalence classes, described by

$$
\left[\gamma_{1}\right],\left[\gamma_{2}\right],\left[\gamma_{4}\right],\left[\gamma_{5}\right]
$$

With Prop. 1, this implies that each of the correspondences $e_{R A T}, e_{d C E}$, and $e_{N E}$ distinguishes at most the equivalence classes $\left[\gamma_{1}\right],\left[\gamma_{2}\right],\left[\gamma_{4}\right],\left[\gamma_{5}\right]$. The next proposition provides a precise characterization.

Proposition 2 For the $2 \times 2$ two-player games, the rationalizable, correlated, and Nash equivalence classes all coincide and distinguish exactly three classes of games, which are described by

$$
\left[\gamma_{1}, \gamma_{2}\right],\left[\gamma_{4}\right],\left[\gamma_{5}\right]
$$

Proof. It follows directly from Prop. 1 that there are at most four rationalizable, correlated and Nash equivalence classes, since all games within the corresponding nondegenerate equivalence classes $\left[\gamma_{1}\right],\left[\gamma_{2}\right],\left[\gamma_{4}\right]$, and $\left[\gamma_{5}\right]$ are also equivalent with respect to these notions. Moreover, it is easy to check that the games $\gamma_{1}, \gamma_{2}$ can all be connected by paths of games along which $e_{R A T}, e_{N E}$, and $e_{d C E}$ are all continuous, so that they are also rationalizable, correlated and Nash equivalent. This means that, at most, there are three classes $\left[\gamma_{1}, \gamma_{2}\right],\left[\gamma_{4}\right]$, and $\left[\gamma_{5}\right]$ of rationalizable, correlated and Nash equivalent games.

Next we show that for each of the three equivalence concepts there are in fact exactly these three equivalence classes. We start by showing it for Nash equivalence and show $\gamma_{1} \chi_{N E} \gamma_{4}, \gamma_{1} \chi_{N E} \gamma_{5}$, and $\gamma_{4} \chi_{N E} \gamma_{5}$. This immediately implies corresponding statements about correlated and rationalizable equivalence.

The proof is geometric. Take the game $\gamma_{4}$. It has a unique Nash equilibrium in mixed strategies and is depicted in Figure 1a below together with the players' best-reply correspondences. Any game in the neighborhood has similar best-reply correspondences, which may be slightly shifted in direction of the arrows on the figure, parallel to the axes. Shifting the best-reply correspondences sufficiently will eventually lead to a game with a continuum of Nash equilibria such as the game

$$
\gamma_{41}=\left(\begin{array}{ll}
\left(1, \frac{1}{2}\right) & \left(\frac{1}{2}, \frac{1}{2}\right) \\
(0,1) & \left(\frac{1}{2}, 0\right)
\end{array}\right)
$$


which is exactly halfway between $\gamma_{4}$ and $\gamma_{1}$ and which is depicted in Figure $1 \mathrm{~b}$. At such a game the correspondence $e_{N E}$ is clearly discontinuous. Similarly, it can be checked that games from the class $\left[\gamma_{4}\right]$ (and $\left[\gamma_{1}\right]$ ) have a unique correlated equilibrium and hence the dimension jumps from zero to one when reaching games like $\gamma_{41}$ and it is clear from Figure 1a that such jumps are unavoidable. This shows $\gamma_{1} \chi_{N E} \gamma_{4}$ and $\gamma_{1} \chi_{C E} \gamma_{4}$, as well as $\gamma_{4} \chi_{N E} \gamma_{5}$ and $\gamma_{4} \chi_{C E} \gamma_{5}$. Obviously, $\gamma_{1} \chi_{R A T} \gamma_{4}$ since the number of strategies per player surviving iterated strict dominance is respectly one and two for the two games. To see $\gamma_{4} \chi_{R A T} \gamma_{5}$ is somewhat more suttle. Both games $\gamma_{4}$ and $\gamma_{5}$ have the same number of rationalizable strategies, however it is not difficult to see that it is impossible to join the two games along a path where $e_{R A T}$ is everywhere continuous. Discontinuities at games between the two classes are unavoidable, since, in any arbitrarily small neighborhood around such games, $e_{R A T}$ goes from containing all strategies to just containing one strategy profile. (Notice, however, that this does not mean that a path between the two classes along which the number of rationalizable strategies is constant does not exist; as for example the path through the game where all entries are zero.)

\section{Figure 1}

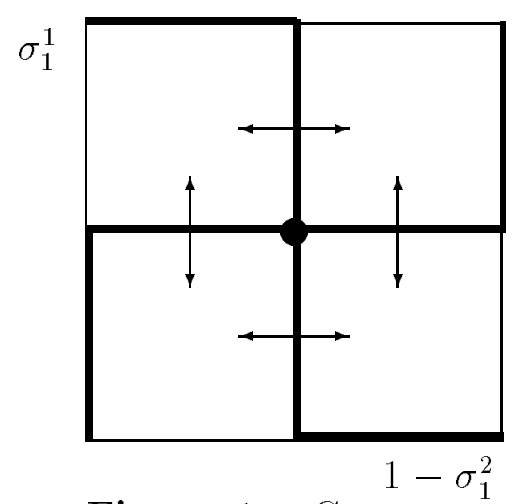

Figure 1a. Game $\gamma_{4}$

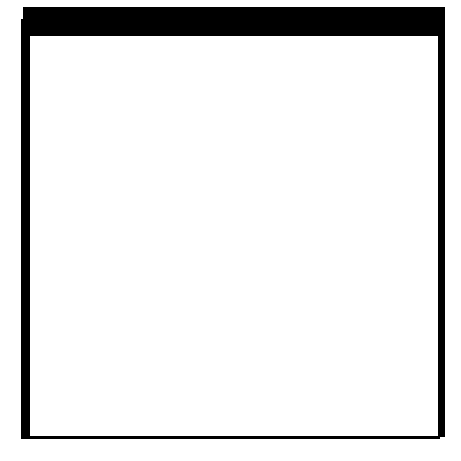

Figure 1b. Game $\gamma_{41}$

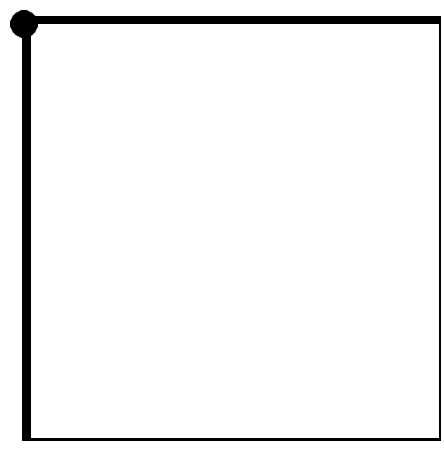

Figure 1c. Game $\gamma_{1}$

Next, take the game $\gamma_{5}$. It has a unique mixed strategy equilibrium and two pure strategy equilibria and is also depicted in Figure 2a along with the best-reply correspondences. Again, the arrows show possible (parallel) shifts of the best-reply correspondences for neighboring games. Also here one sees that whatever the direction of the shifts, it is impossible to leave the class of games $\left[\gamma_{5}\right]$ without encountering either a game with a continuum 
of Nash equilibria or games like the game

$$
\gamma_{51}=\left(\begin{array}{ll}
(1,1) & \left(\frac{1}{2}, 0\right) \\
\left(0, \frac{1}{2}\right) & \left(\frac{1}{2}, \frac{1}{2}\right)
\end{array}\right)
$$

which is exactly halfway between $\gamma_{5}$ and $\gamma_{1}$ and which is depicted in Figure $2 \mathrm{~b}$. This game has exactly two Nash equilibria, one of which (the one on the lower-right corner) disappears as the payoffs further change towards the payoffs of $\gamma_{1}$. This implies $\gamma_{1} \chi_{N E} \gamma_{5}$ since, at such a game, the correspondence $e_{N E}$ is not continuous.

\section{Figure 2}

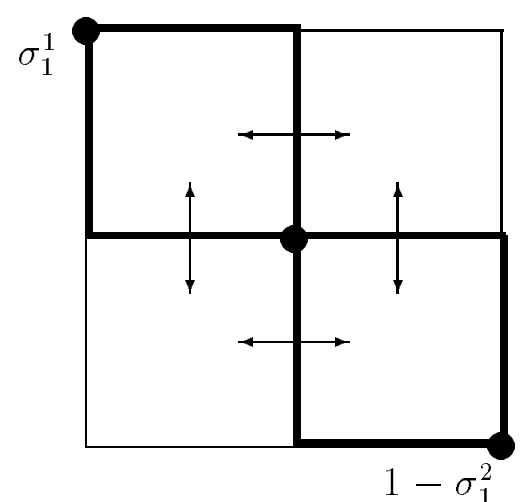

Figure 2a. Game $\gamma_{5}$

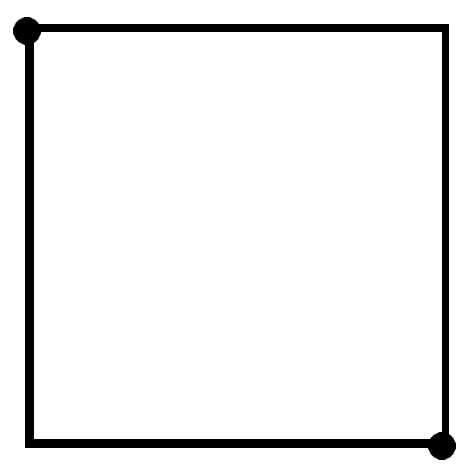

Figure 2b. Game $\gamma_{51}$

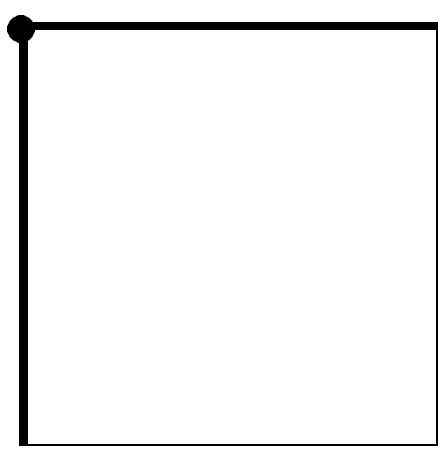

Figure 2c. Game $\gamma_{1}$

On the other hand, the games $\gamma_{5}$ and $\gamma_{1}$ cannot be correlated equivalent, since the dimensions of the corresponding sets of correlated equilibria are three and zero respectively. Similarly, they cannot be rationalizable equivalent, since again the number of strategies per player surviving iterated strict dominance is respectively two and one. This completes the proof.

Somewhat surprisingly, the equivalence classes for the three standard concepts of rationalizability, correlated and Nash equilibrium all coincide. The equivalence classes consist of games with only one pure Nash equilibrium, $\left[\gamma_{1}, \gamma_{2}\right]$, games with only one mixed Nash equilibrium, $\left[\gamma_{4}\right]$, and games with one mixed and two pure Nash equilibria, $\left[\gamma_{5}\right]$. Examples of games in the different classes are the prisoners' dilemma games and other strict dominance solvable games for $\left[\gamma_{1}, \gamma_{2}\right]$, matching pennies games for $\left[\gamma_{4}\right]$, and games like battle of the sexes, chicken, and other coordination games for $\left[\gamma_{5}\right]$. 
In particular, the classes $\left[\gamma_{4}\right]$ and $\left[\gamma_{5}\right]$ which both have all strategies being rationalizable, are nonetheless not rationalizable equivalent. Similarly, all games in the classes $\left[\gamma_{1}, \gamma_{2}\right]$ and $\left[\gamma_{4}\right]$, have a uique correlated and a unique Nash equilibrium, yet are neither correlated nor Nash equivalent. Showing these two facts constitute the main part of the proof; an important step in this regard consists in showing that discontinuities of all three correspondences $e_{R A T}, e_{C E}$, and $e_{N E}$ are inevitable when leaving the class $\left[\gamma_{4}\right]$. This also shows that simply counting the number of rationalizable strategies or of Nash (or correlated) equilibria is not sufficient to obtain the corresponding classification. We will come back to $2 \times 2$ games further below in the context of robust and risk dominant equilibria, where we also briefly discuss some experimental literature.

\subsection{Larger Games}

We briefly discuss games larger than $2 \times 2$ and show that the characterizations for the rationalizable, correlated, and Nash equivalence classes no longer coincide for larger games.

It can be shown that the space of $2 \times 3$ two-player games is divided by the singular games into 336 connected components of nondegenerate games, which after applying the symmetry operations reduce to 28 nondegenerate equivalence classes. The equivalence classes for the $2 \times 3$ games are all different across the three mentioned standard equilibrium concepts. It can be shown that there are four distinct rationalizable equivalence classes consisting of a class represented by games with one strategy per player surviving iterated strict dominance, two classes of games with two strategies per player surviving iterated strict dominance, and games with all strategy profiles surviving iterated strict dominance. Examples of the latter games are

$$
\gamma_{6}=\left(\begin{array}{lll}
(1,3) & (1,2) & (0,0) \\
(0,0) & (0,2) & (1,3)
\end{array}\right), \gamma_{7}=\left(\begin{array}{lll}
(1,3) & (0,2) & (1,0) \\
(0,0) & (1,2) & (0,3)
\end{array}\right)
$$

which satisfy $\gamma_{6} \sim_{R A T} \gamma_{7}$. It can further be shown that there are also four distinct correlated equivalence classes represented by the games $\gamma_{1}^{\prime}, \gamma_{4}^{\prime}$, and $\gamma_{5}^{\prime}$, obtained from $\gamma_{1}, \gamma_{4}, \gamma_{5}$ by adding a strictly dominated strategy for player 2 to make them $2 \times 3$ games, and games like $\gamma_{6}$ or $\gamma_{7}$ which can be shown to also satisfy $\gamma_{6} \sim_{C E} \gamma_{7}$, and where the dimension of the set of correlated equilibria is five for both games. Finally, it can be shown that there are five distinct Nash equivalence classes represented by the games $\gamma_{1}^{\prime}, \gamma_{4}^{\prime}, \gamma_{5}^{\prime}, \gamma_{6}$, and $\gamma_{7}$, which can all be shown not to be Nash equivalent. The latter two are representatives of games with two pure strategy and 
one mixed strategy equilibria, $\gamma_{6}$, where all pure strategy profiles are in the support of some Nash equilibrium, and of games with with one pure strategy and two mixed strategy equilibria, $\gamma_{7}$.

Hence, unlike the case of $2 \times 2$ games, the classifications for the three standard equivalence concepts are all different in the $2 \times 3$ games and can be summarized as follows. There are four distinct rationalizable equivalence classes described by

$$
\left[\gamma_{1}^{\prime}, \gamma_{2}^{\prime}, \ldots\right],\left[\gamma_{4}^{\prime}, \ldots\right],\left[\gamma_{5}^{\prime}, \ldots\right],\left[\gamma_{4}^{\prime \prime}, \gamma_{6}, \gamma_{7}\right]
$$

there are also four distinct correlated equivalence classes, which slightly differ from the rationalizable equivalence classes, and are described by

$$
\left[\gamma_{1}^{\prime}, \gamma_{2}^{\prime}, \ldots\right],\left[\gamma_{4}^{\prime}, \gamma_{4}^{\prime \prime}, \ldots\right],\left[\gamma_{5}^{\prime}, \ldots\right],\left[\gamma_{6}, \gamma_{7}\right]
$$

and there are five distinct Nash equivalence classes described by

$$
\left[\gamma_{1}^{\prime}, \gamma_{2}^{\prime}, \ldots\right],\left[\gamma_{4}^{\prime}, \gamma_{4}^{\prime \prime}, \ldots\right],\left[\gamma_{5}^{\prime}, \ldots\right],\left[\gamma_{6}\right],\left[\gamma_{7}\right]
$$

For reasons of space and since they can be unambiguously determined from the mentioned set of 28 distinct nondegenerate equivalence classes, we have not listed all the representatives of the nondegenerate equivalence classes and have instead referred to them by the dots. The nondegenerate equivalence class $\left[\gamma_{4}^{\prime \prime}\right]$ is what distinguishes the rationalizable and the correlated equivalence classes, while the nondegenerate equivalence classes $\left[\gamma_{6}\right]$ and $\left[\gamma_{7}\right]$ are the ones distinguishing the Nash and the correlated equivalence classes. The games $\gamma_{4}^{\prime}$ and $\gamma_{4}^{\prime \prime}$ are discussed below.

Notice that within the nondegenerate $2 \times 2$ and $2 \times 3$ games, although knowing the number of Nash equilibria $\left|e_{N E}(\gamma)\right|$ is not sufficient, knowing the Nash equilibrium distributions, i.e., knowing $e_{N E}(\gamma)$, is sufficient to place the game $\gamma$ unambiguously within its Nash equivalence class. The latter also fails with the $3 \times 3$ games as is illustrated by the following two games

$$
\gamma_{8}=\left(\begin{array}{ccc}
(5,3) & (0,0) & (5,2) \\
(4,3) & (2,2) & (2,0) \\
(0,0) & (5,2) & (0,3)
\end{array}\right), \gamma_{9}=\left(\begin{array}{lll}
(5,3) & (0,0) & (5,2) \\
(0,3) & (5,2) & (0,0) \\
(4,0) & (2,2) & (2,3)
\end{array}\right)
$$

Both games have the same Nash equilibria, namely the pure strategy equilibrium, where both play strategy 1, the mixed strategy equilibrium where players 1 and 2 mix between respectively strategies 2 and 3 , and 1 and 2 , and, finally, the completely mixed equilibrium. To understand why the two 
games are not Nash equivalent, one could for example compare the indices of the mixed strategy equilibria. However, it can be shown that having the same Nash equilibrium distributions with same indices is also not sufficient to imply Nash equivalence of the games, thus implying that besides the index, something like the orientation of a given (monotonic) dynamics may matter in determining the Nash equivalence class; see Germano (1998) for further discussion and definitions. On the other hand, already within the $2 \times 2$ games, knowing $e_{R A T}(\gamma)$ or $e_{d C E}(\gamma)$ was not sufficient to place $\gamma$ in its respective equivalence class, as the games $\gamma_{4}$ and $\gamma_{5}$ showed for rationalizable equivalence and the games $\gamma_{1}$ and $\gamma_{4}$ showed for correlated equivalence.

Although the classification of the previous section provides some insight concerning the games distinguished by the given equilibrium concepts, one should not deduce from the $2 \times 2$ case, that two games that are Nash or correlated equivalent (or both) are also necessarily rationalizable equivalent, as the following $2 \times 3$ matching pennies type games show

$$
\gamma_{4}^{\prime}=\left(\begin{array}{ccc}
(1,0) & (0,1) & (2,-2) \\
(0,1) & (1,0) & (-2,-2)
\end{array}\right), \gamma_{4}^{\prime \prime}=\left(\begin{array}{lll}
(1,0) & (0,1) & (2,-2) \\
(0,1) & (1,0) & (-2,2)
\end{array}\right) .
$$

Both $\gamma_{4}^{\prime}$ and $\gamma_{4}^{\prime \prime}$ have a the same unique Nash and correlated equilibrium (involving only the first two strategies) and are clearly Nash and correlated equivlent. However, the set of rationalizable strategies changes, as player 2 's third strategy is no longer strictly dominated in $\gamma_{4}^{\prime \prime}$.

Similarly, two games that are rationalizable or correlated (or both) need not be Nash equivalent, as the games $\gamma_{6}$ and $\gamma_{7}$ above show.

Furthermore, the games $\gamma_{8}$ and $\gamma_{9}$ show that two games that are rationalizable equivalent need not be correlated equivalent, and the following example shows that two games that are Nash equivalent need not be correlated equivalent

$$
\gamma_{10}=\left(\begin{array}{lll}
(5,3) & (5,-1) & (0,0) \\
(2,2) & (2,-1) & (4,3) \\
(0,0) & (0,-1) & (5,3)
\end{array}\right), \gamma_{11}=\left(\begin{array}{lll}
(5,3) & (5,2) & (0,0) \\
(2,2) & (2,0) & (4,3) \\
(0,0) & (0,2) & (5,3)
\end{array}\right)
$$

Both games have the same three Nash equilibria, two pure where both players play strategies 1 and 3 , and a mixed equilibrium where they mix respectively between strategies 1 and 2 and 1 and 3 . Notice that in neither of the games is player 2 's strategy 2 used, and, in $\gamma_{11}$ it is actually strictly dominated. While moving from game $\gamma_{10}$ to $\gamma_{11}$ does not affect the Nash equilibrium correspondence, the dimension of the set of correlated equilibria is five at $\gamma_{10}$ and eight at $\gamma_{11}$. 
To summarize then, unlike the $2 \times 2$ case, where the three equivalence notions coincide, it is possible, already within the $3 \times 3$ games, to find examples showing that Nash or correlated equivalence (or both) need not imply rationalizable equivalence; that rationalizable or correlated equivalence (or both) need not imply Nash equivalence; and that Nash or rationalizable equivalence need not imply correlated equivalence. ${ }^{8}$ However, we found one notion, nondegenerate equivalence, that, at least for two player games, unifies the three equivalence notions (see Prop. 1).

\section{Robust Equilibria and Risk Dominance}

We consider a fourth equilibrium concept, namely that of robust equilibrium of Kajii and Morris $(1997 \mathrm{a}, \mathrm{b}) .{ }^{9}$ The equilibrium concept involves consideration of neighboring incomplete information games. Let $T_{i}^{s}$ denote a countable set of standard types of player $i$, and let $T_{i}^{c}=S_{i}$ denote the set of committed types of player $i$; the set of all possible types of player $i$ is $T_{i}=T_{i}^{s} \cup T_{i}^{c}$, let also $T=\times_{i \in I} T_{i}$ and $T_{-i}=\times_{j \neq i} T_{j}$. Notice that a committed (or "crazy") type of player $i$ of type $t_{i}$ will have a strictly dominated strategy to play pure strategy $t_{i}$. From a complete information game $\gamma \in \mathbb{R}^{\kappa n}$ and a probability distribution over types $P \in \Delta(T)$, we can derive an incomplete information game with payoffs

$$
g^{i}(s ; t)=\left\{\begin{array}{cl}
\gamma^{i}(s) & \text { if } t^{i} \in T_{i}^{s} \\
1 & \text { if } s^{i}=t^{i} \in T_{i}^{c} \\
0 & \text { if } s^{i} \neq t^{i}, t^{i} \in T_{i}^{c},
\end{array}\right.
$$

for $s \in S, t \in T$, and $i \in I$. The following defines incomplete information games that are $\epsilon$-close to the complete information game $\gamma$.

Definition 3 A canonical $\epsilon$-elaboration consists of a complete information game $\gamma$ and a probability distribution $P \in \Delta(T)$ such that $P\left[T^{s}\right]=1-\epsilon$ for $\epsilon \in[0,1]$. A strategy profile $\sigma \in \Sigma$ is robust to all canonical elaborations if, $\forall \delta>0, \exists \bar{\epsilon}>0$ such that, $\forall \epsilon \leq \bar{\epsilon}$, every $\epsilon$-elaboration of $\gamma$ has a Bayesian Nash equilibrium $\mu$ with $\mu(\sigma) \geq 1-\delta$. Finally, we say a strategy profile is a robust equilibrium if it is robust to all canonical elaborations.

\footnotetext{
${ }^{8}$ We do not have an example of two games that are at the same time Nash and rationalizable equivalent but not correlated equivalent. Thus, so far, we cannot exclude that the intersection of the equivalence classes of the first two concepts may contain the correlated equivalence classes.

${ }^{9}$ Carlsson and van Damme (1993), Morris et al. (1995) are related approaches for two player games, see also Morris and Shin (2003) for further related literature; Cabrales et al. (2002) provide experimental evidence.
} 
Again with Def. 1, we say that two games $\gamma_{0}, \gamma_{1} \in \mathbb{R}^{\kappa n}$ are robust equivalent, which we denote by $\gamma_{0} \sim_{R E} \gamma_{1}$, if they are equivalent with respect to $e_{R E}$, where $e_{R E}$ is the robust equilibrium correspondence.

To get some intuition about robust equivalence classes, consider the $2 \times 2$ games. The analog of Prop. 1 does not hold for robust equivalence. In fact, it can be shown that the correspondence $e_{R E}$ is not always continuous at nondegenerate games. To see this, notice that it follows from Kajii and Morris (1997b) that, for $2 \times 2$ games with two strict equilibria, the robust equilibria coincide with the risk dominant equilibria (see Harsanyi and Selten (1988) or van Damme (2002)). One implication of this is that there is a set $\Upsilon$ of nondegenerate games in $\left[\gamma_{5}\right]$ at which the robust equilibrium correspondence is not continuous, namely,

$$
\Upsilon=\left\{\begin{array}{l|l}
\gamma \in\left[\gamma_{5}\right] & \begin{array}{l}
\left(\gamma_{11}^{1}-\gamma_{21}^{1}\right)\left(\gamma_{11}^{2}-\gamma_{12}^{2}\right)=\left(\gamma_{22}^{1}-\gamma_{12}^{1}\right)\left(\gamma_{22}^{2}-\gamma_{21}^{2}\right) \text { if } \gamma_{11}^{1}>\gamma_{21}^{1} \\
\left(\gamma_{12}^{1}-\gamma_{22}^{1}\right)\left(\gamma_{12}^{2}-\gamma_{11}^{2}\right)=\left(\gamma_{21}^{1}-\gamma_{11}^{1}\right)\left(\gamma_{21}^{2}-\gamma_{22}^{2}\right) \text { if } \gamma_{11}^{1}<\gamma_{21}^{1}
\end{array}
\end{array}\right\} .
$$

These are precisely games in $\left[\gamma_{5}\right]$ where the deviation losses for the two (strict) equilibria are equal. An example of such a game is the game $\gamma_{5}$ itself; it is easy to see that with arbitrarily small variations of the payoffs, the robust equilibrium moves from the top-left to the bottom-right strategy profile. Furthermore, Kajii and Morris (1997b) have also shown that nondegenerate games in $\left[\gamma_{1}\right],\left[\gamma_{2}\right]$, and $\left[\gamma_{4}\right]$ all have a unique robust equilibrium which is also the unique Nash equilibrium. We summarize this as follows.

Lemma 4 Every nondegenerate $2 \times 2$ two-player game not in $\Upsilon$ has a unique robust equilibrium, which coincides with the unique risk dominant Nash equilibrium of the game.

Now, we can state the characterization of the robust equivalence classes for $2 \times 2$ games.

Proposition 3 For the $2 \times 2$ two-player games, there are two distinct robust equivalence classes described by

$$
\left[\gamma_{1}, \gamma_{2}, \gamma_{5}\right] \backslash \Upsilon \text { and }\left[\gamma_{4}\right]
$$

Proof. The proof is in two steps. First, we show that any two games in $\left[\gamma_{1}, \gamma_{2}, \gamma_{5}\right] \backslash \Upsilon$ as well as in $\left[\gamma_{4}\right]$ are robust equivalent. Second, we show that games from the two different classes are not robust equivalent.

Clearly, all games in $\left[\gamma_{1}, \gamma_{2}\right]$ are robust equivalent, and the same holds for games in $\left[\gamma_{4}\right]$. Similarly, despite the discontinuity at $\Upsilon$, all games in $\left[\gamma_{5}\right] \backslash \Upsilon$ are robust equivalent, due to symmetry operations identifying games with 
strict equilibria at the top-left and bottom-right profiles with corresponding games with strict equilibria at the bottom-left and top-right profiles. Finally, to see equivalence of a game from $\left[\gamma_{1}, \gamma_{2}\right]$ with a game from $\left[\gamma_{5}\right] \backslash \Upsilon$, consider the following game

$$
\hat{\gamma}_{\epsilon}=\left(\begin{array}{ll}
(1,1) & (0,0) \\
(0,0) & (\epsilon, \epsilon)
\end{array}\right),
$$

where $\epsilon<1$. For $\epsilon \in(0,1)$, we have $\hat{\gamma}_{\epsilon} \in\left[\gamma_{5}\right] \backslash \Upsilon$, while for $\epsilon<0$, we have $\hat{\gamma}_{\epsilon} \in\left[\gamma_{1}, \gamma_{2}\right]$. In particular, we have that $\hat{\gamma}_{\frac{1}{2}} \in\left[\gamma_{5}\right] \backslash \Upsilon$ and $\hat{\gamma}_{-\frac{1}{2}} \in\left[\gamma_{1}, \gamma_{2}\right]$. On the other hand, for all $\epsilon<1$, the game $\hat{\gamma}_{\epsilon}^{2}$ has a unique robust equilibrium at the top-left strategy profile, and, moreover, the correspondence $e_{R E}$ is continuous at any such game. This shows in particular $\hat{\gamma}_{\frac{1}{2}} \sim_{R E} \hat{\gamma}_{-\frac{1}{2}}$ and hence the first step.

To see the second step, cosider the game

$$
\bar{\gamma}_{\epsilon}=\left(\begin{array}{ll}
(1,0) & (0, \epsilon) \\
(0,1) & (\epsilon, 0)
\end{array}\right),
$$

where again $\epsilon<1$. For $\epsilon \in(0,1)$, we have $\bar{\gamma}_{\epsilon} \in\left[\gamma_{4}\right]$, while for $\epsilon<0$, we have $\bar{\gamma}_{\epsilon} \in\left[\gamma_{1}, \gamma_{2}, \gamma_{5}\right] \backslash \Upsilon$. However, as can be seen in Figure 3 , and analogous to the reasoning in the proof of Prop. 2, discontinuities of $e_{R E}$ are unavoidable when going from games in $\left[\gamma_{4}\right]$ to games in $\left[\gamma_{1}, \gamma_{2}, \gamma_{5}\right] \backslash \Upsilon$.

\section{Figure 3}

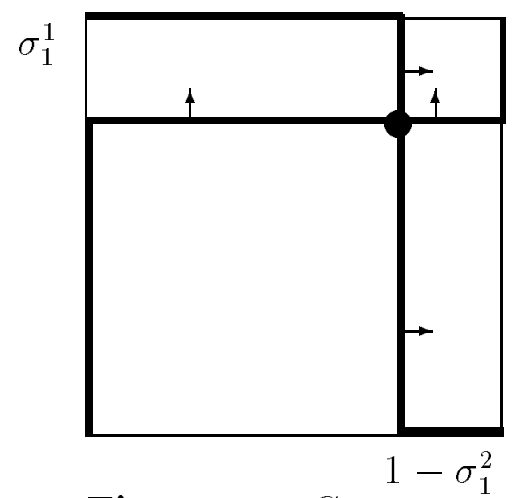

Figure 3a. Game $\bar{\gamma}_{\frac{1}{3}}$

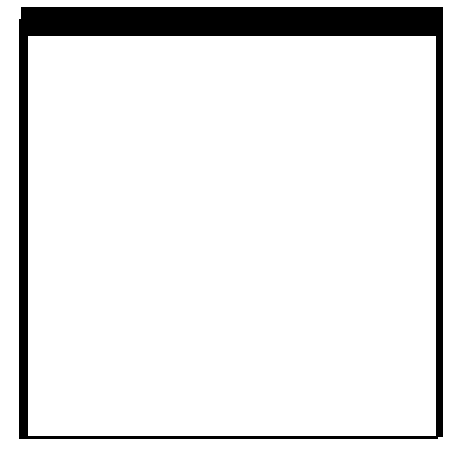

Figure 3b. Game $\bar{\gamma}_{0}$

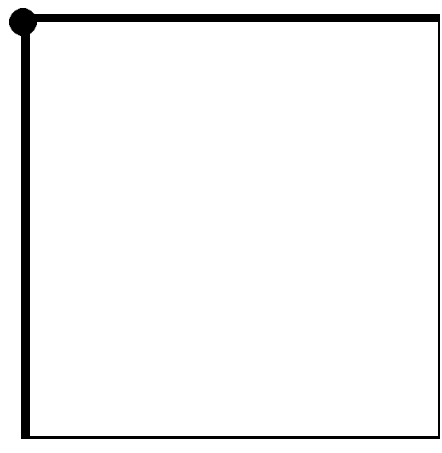

Figure 3c. Game $\bar{\gamma}_{-\frac{1}{3}}$

(Notice that the bold dots in Figure 3 indicate the (unique) robust equilibria.) This shows that games in $\left[\gamma_{4}\right]$ are not robust equivalent to games in $\left[\gamma_{1}, \gamma_{2}, \gamma_{5}\right] \backslash \Upsilon$ and so completes the proof. 
Robust equivalence identifes games in $\left[\gamma_{5}\right] \backslash \Upsilon$ with games in $\left[\gamma_{1}, \gamma_{2}\right]$, i.e., games with two pure Nash equilibria with games with unique pure Nash equilibrium. We view this as a fundamental departure from the equivalence classes obtained in the previous section, also since most casual discussions or other classifications in the literature typically distinguish the two classes of games, see, e.g., Rapoport et al. (1976), Carlsson and van Damme (1993), Roth (1995), or Friedman (1996).

Moreover, we view experimental evidence as providing support for the robust equivalence classes in the following sense. Take the game $\hat{\gamma}_{\epsilon}$ of Eq. (1) and suppose that $\epsilon$ is arbitrarily close to zero (units could be millions of euros). In this case, while it matters for rationalizable, correlated, and Nash equivalence, whether $\epsilon$ is positive or negative, (since a discontinuity occurs at $\epsilon=0$ ), it does not matter for robust equivalence, as long as $\epsilon$ is sufficiently small; further, when $\epsilon$ is equal to 1 , then a discontinuity of the set of robust equilibria occurs, (since $\hat{\gamma}_{1}$ is in $\Upsilon$ ), while each of the rationalizable, correlated, and Nash correspondences are all continuous at such a game. In both cases, experimental evidence seems to be better explained by the predictions of the robust equilibria; because of the usual risk dominance argument for $\epsilon$ close to 0 , and because of the issue of which strategy profile to coordinate on for $\epsilon$ close to 1; see Ochs (1995), Straub (1995), Friedman (1996), Crawford (1997), for experimental evidence, and also Cabrales et al. (2002), who test further connections with global games.

At the same time, all four equilibrium concepts identify all games in $\left[\gamma_{1}, \gamma_{2}\right]$ as well as all games in $\left[\gamma_{5}\right]$ or $\left[\gamma_{5}\right] \backslash \Upsilon$. In particular, this means that, for games in $\left[\gamma_{1}, \gamma_{2}\right]$, whether the (unique) profile surviving iterated dominance, which is also the unique Nash, correlated and robust equilibrium is also a Pareto efficient outcome or not, is irrelevant to the equilibrium concepts in the sense of the induced equivalence classes; on the other hand, there is experimental evidence suggesting that at least some extra variance is observed when the unique equilibrium point does not coincide with the Pareto efficient outcome, as in the prisoners' dilemma; see, e.g., Rapoport et al. (1976) and Roth (1995), and Ahn et al. (2002) for some experiments on such games. Similarly, for games in $\left[\gamma_{5}\right]$ or $\left[\gamma_{5}\right] \backslash \Upsilon$, whether or not the risk dominant equilibrium is also Pareto efficient or whether and what type of a battle of the sexes game it is, although not relevant to the studied equivalence classes, seems to matter experimentally; see, e.g., Ochs (1995), Crawford (1997), Battalio et al. (2001), and van Huyck and Battalio (2002). A variable that may be worth considering when testing experimental behavior is a measure of the distance of the given game to the next closest discontinuity of the relevant equilibrium correspondence under study. Finally, the class $\left[\gamma_{4}\right]$, which is also distinguished by all four concepts, appears 
to exhibit relatively more homogenous behavior; see Erev and Roth (1998) for experimental evidence on such games as well as further references.

\section{$5 \quad$ Related Literature}

Rapoport et al. (1976) consider ordinal $2 \times 2$ two player games which, after distinguishing 78 strategically different games, they classify into 24 different classes. Their distinctions are based on several notions, including whether the "natural outcome" (this is the intersection of the maxmin profiles) is a Nash equilibrium, whether it is Pareto efficient, or what kinds of threats the players can exercise against each other. In particular, the following three (coordination type) games all belong to different classes,

$$
\gamma_{61}=\left(\begin{array}{ll}
(4,4) & (1,3) \\
(3,1) & (2,2)
\end{array}\right), \gamma_{64}=\left(\begin{array}{ll}
(3,4) & (2,1) \\
(1,2) & (4,3)
\end{array}\right), \gamma_{68}=\left(\begin{array}{ll}
(3,4) & (2,2) \\
(1,1) & (4,3)
\end{array}\right)
$$

where the indices here correspond to the labels of Rapoport et al. (1976). As Bárány et al. (1992) point out, it is not clear how their procedure extends to larger games, since certain parts of their classification are defined specifically for the $2 \times 2$ games. Their analysis has a very strong experimental motivation, which may explain the fact that their distinctions are not necessarily related to the standard non-cooperative equilibrium concepts. Rapoport and Guyer (1966) and Harris (1972) study related distinctions; see also Rapoport et al. (1976) for further related references.

Bárány et al. (1992) take a combinatorial approach and classify $m \times n$ two player ordinal games. They also start from the set of strategically different (ordinal) games and look at the numbers of possible outcome sets generated by the payoff matrices, as well as the numbers of shapes of the corresponding convex hulls. They then derive asymptotic estimates for the number of strategically different games $\left(\approx(m n)^{2 m n}\right)$, for the number of different outcome sets $\left(\approx(m n)^{m n}\right)$, and for the number of different convex hulls of the outcome sets $\left(\approx e^{(m n)^{2 / 3}}\right)$. In the case of $2 \times 2$ two player games, they start from the 78 strategically different games and obtain 24 different outcome sets and 7 different shapes of the convex hulls. In particular, the games $\gamma_{61}, \gamma_{64}, \gamma_{68}$ also belong to different outcome sets which also have different shapes; while on the other hand, the following four games,

$$
\left(\begin{array}{ll}
(4,4) & (3,3) \\
(2,2) & (1,1)
\end{array}\right),\left(\begin{array}{ll}
(3,4) & (2,1) \\
(1,2) & (4,3)
\end{array}\right),\left(\begin{array}{ll}
(2,3) & (4,1) \\
(1,4) & (3,2)
\end{array}\right),\left(\begin{array}{ll}
(2,3) & (4,1) \\
(3,2) & (1,4)
\end{array}\right)
$$

all have the same shape of the convex hull, and the first two have the same outcome set, which is different from the outcome set of the last two. (Notice 
that, while the outcomes for all four games lie on a straight line, the first two are coordination games, the third is a dominance solvable game and the last a matching pennies type game; they correspond to games under Fig. 1 and Fig. 24 in the appendix of Bárány et al. (1992).) Although Bárány et al. also mention the possibility of using properties of the best reply or other non-cooperative equilibrium correspondences to derive alternative classifications, it is clear from the games above that their classification is not really related to the ones implied by the (non-cooperative) equilibrium concepts of this paper. It should also be pointed out that, already for the $3 \times$ 3 games, the number of strategically different ordinal games, which are the basis for the equivalence classes of Bárány et al. (1992) (and also Rapoport et al. (1976)), amounts to over 65 billion games (see Bárány et al. (1992), p. 280). In contrast, there are just over 110,000 different path components separated by the degenerate games, which after applying the symmetry operations reduce to just above 530 different nondegenerate equivalence classes, which further reduce to 32 different Nash equivalence classes for the $3 \times 3$ case (see Germano (1998)).

Within the mathematics-combinatorics literature, Conway (2001) shows how equivalence classes of games can have a nice mathematical structure. He considers the class of so-called nim-type games, (these are two person zero sum games where agents make sequential moves, one after the other, choosing from a given set of strategies that decreases as they make moves), and shows that the set of equivalence classes of these nim-type games can be identified with the space of nonstandard or surreal numbers. Although the class of nim-type games does not seem to be of particular relevance to the social sciences, its classification shows that equivalence classes of games may correspond to interesting mathematical objects. (For further literature on nim-type and related games see also Guy (1991) and especially Conway (2001) and Berlekamp et al. (2003).)

Partly motivated by literature aiming to extend certain results from potential games to larger classes of games, Morris and Ui (2002) define and characterize notions of best-reply, better-reply, and von Neumann and Morgenstern equivalence. These are increasingly restrictive equivalence notions, and the weakest one, best-reply equivalence, which obtains when two games have the same best-reply correspondence, is already a substantially stronger notion than our notion of nondegenerate equivalence. Already within $2 \times 2$ games, best-reply equivalence distinguishes uncountably many matching pennies and coordination games. (See Morris and Ui (2002) for related literature and applications.)

Also related are the notions of analogy or similarity, typically studied within the context of decision situations. Besides an extensive literature in 
psychology on analogical and case-based reasoning, see for example Holyoak and Thagard (1995) and references there, related work has also been done in the economics literature. Rubinstein (1988) defines a notion of similarity, which he uses to define rules of thumb via a procedural rationality approach. Gilboa and Schmeidler (1995) formulate a case-based decision theory which, in a general way, allows decision makers to make use of analogies when assessing the performance of different strategies. Samuelson (2001) models analogies as part of a model which studies the consequences of individuals economizing on their reasoning resources; van Huyck and Battalio (2002) test whether experimental subjects use certain rules to play similar, but different, coordination games; Jehiel (2002) develops an equilibrium concept, analogy-based expectation equilibrium, which is also based on agents using analogies to obtain simplified representations of games and of reactions of their opponents.

\section{Conclusion}

The present paper has introduced a procedure for identifying games into broad equivalence classes of normal form games through the geometries of equilibrium correspondences. The procedure was seen to lead to an organization of the spaces of games into typically finitely many well-defined equivalence classes, which constitute a classification of normal form games relative to given equilibrium concepts. To a certain extent, the classifications obtained reflect important aspects of the logic of the underlying equilibrium concepts, and we find the procedure as providing a useful way of both understanding and comparing different equilibrium concepts, but also of thinking about strategic situations.

Moreover, it points to an alternative method of testing equilibrium theories experimentally, namely, by locating the discontinuities implied by equilibrium concepts and testing actual behavior directly for the discontinuities. It is also possible that the location of a game within an equivalence class provides relevant information and that a measure of distance of a given game to the nearest discontinuity of the underlying equilibrium correspondence may prove a useful variable for explaining experimentally observed behavior.

At the same time, many questions are left unanswered. First, understanding the structure of the set of equivalence classes for Nash, correlated, or robust equilibria for a given space of games, and to see how these change when strategies and/or players are added is not an easy task. Our analysis focused on $2 \times 2$ games and it seems worthwhile studying larger games. It 
would also be interesting to obtain a clearer understanding of general relationships between the rationalizable, correlated, Nash, and robust equivalence classes. The (stronger) notion of nondegenerate equivalence was seen to provide a first basic link.

On the other hand, a somewhat unsatisfactory aspect of the present analysis is precisely that, while it leads to finitely many equivalence classes, as long as the equilibrium correspondence is semi-algebraic, the number of classes distinguished seems to increase very rapidly with the number of players and/or strategies. For example, McKelvey and McLennan (1997) show that games with ten players with two strategies each, can have up to 1.3 million competely mixed (regular) equilibria. (See also Keiding (1997), McLennan (1997), and von Stengel (1999) for more literature on the maximal numbers of Nash equilibria of normal form games.) Although it needs to be checked exactly to what extent such numbers are related to say the number of Nash equivalence classes, it suggests that they could be large.

Finally, something that has not been addressed at all in the present paper, is whether one can use the classifications to obtain insights about degenerate or extensive form games as well as refinements of equilibria. What we have in mind is to look at (standard) equivalence classes around given degenerate or extensive form games in order to understand the different equilibria or components of equilibria at such games. A further possibility would be to consider refinements concepts and define equivalence classes directly on spaces of degenerate or extensive form games. This may also help to gain further insights, e.g., relative to the comparisons in Kajii and Morris (1997b).

In principle, the procedure applies to almost arbitrary spaces of games and equilibrium concepts. An important step, however, would consist in finding ways of defining equivalence classes that are as unrestricted as possible by the specifics of the actual game structure or of the underlying space of games, as, e.g., the notion of a coordination game does not depend on whether the game is a $2 \times 2$ or a $3 \times 3$ game, or whether it is a static or extensive form game. Despite some of its rigidity, we view the present approach as a first step in this direction. An alternative approach, going further in this direction, is Samuelson (2001), who uses automata to characterize agents and in some sense distinguishes fairly different types of games by means of complexity measures.

\section{Appendix}

In this appendix we prove the lemmas and propositions left unproven in the text. 
Proof of Lemma 1. For the sake of completeness, we prove the following more comprehensive statement.

Lemma 5 The correspondences $e_{R A T}, e_{C E}, e_{d C E}, e_{N E}$, and $e_{N D}$ are all semialgebraic and hence there exist closed lower dimensional subsets of the space of games, $D_{R A T}, D_{C E}, D_{d C E}, D_{N E}, D_{N D} \subset \mathbb{R}^{\kappa n}$, such that the correspondences are continuous respectively on the complements $\mathbb{R}^{\kappa n} \backslash D_{R A T}, \mathbb{R}^{\kappa n} \backslash$ $D_{C E}, \mathbb{R}^{\kappa n} \backslash D_{d C E}, \mathbb{R}^{\kappa n} \backslash D_{N E}$, and $\mathbb{R}^{\kappa n} \backslash D_{N D}$. Moreover, the complements all consist of a finite number of connected components.

We only need to check that the correspondences are semi-algebraic; the remaining statements are standard, see, e.g., Schanuel et al. (1991) and Blume and Zame (1994).

(i) $e_{R A T}$ : For all $i \in I$, let $\Sigma_{i}^{0}=\Sigma_{i}$ and recursively define

$$
\Sigma_{i}^{t+1}=\left\{\sigma_{i} \in \Sigma_{i}^{t} \mid \exists \sigma_{-i} \in \times_{j \neq i} \tilde{\Sigma}_{j}^{t}, \forall \sigma_{i}^{\prime} \in \Sigma_{i}^{t} \text {, with } \gamma_{i}\left(\sigma_{i}, \sigma_{-i}\right) \geq \gamma_{i}\left(\sigma_{i}^{\prime}, \sigma_{-i}\right)\right\},
$$

where $\tilde{\Sigma}_{j}^{t}$ is the convex hull of $\Sigma_{j}^{t}$. Then the set of rationalizable strategies of player $i$ is the set $R_{i}=\bigcap_{t=0}^{\infty} \Sigma_{i}^{t}$. However, as Pearce (1984) shows, the elimination procedure ends after a finite number of iterations for finite games, which implies that each $R_{i}$ is a semi-algebraic set and hence so is $e_{R A T}$.

(ii) $e_{C E}$ and $e_{d C E}$ : The case of $e_{C E}$ follows directly from Eq. (2) below, which shows that the set of correlated equilibria is defined by a finite number of linear inequalities. This also implies that $e_{d C E}$ must be semi-algebraic, since computing the dimension of a finite polyhedron is a semi-algebraic operation.

(iii) $e_{N E}$ : This is shown in Schanuel et al. (1991).

(iv) $e_{N D}$ : This follows from the fact that checking whether a game is nondegenerate or not can be reduced to computing determinants of a finite number of matrices, see von Stengel (2002), p. 1740, which are semialgebraic operations.

Proof of Lemma 2. It needs to be shown that the equivalence relation is reflexive, symmetric, and transitive. This is straightforward, see Germano (1998).

Proof of Proposition 1. Let $\gamma_{0}, \gamma_{1} \in \mathbb{R}^{2 \kappa}$ be two two-player games with $\gamma_{0} \sim_{N D} \gamma_{1}$. By definition of $\sim_{N D}$ both games are nondegenrate. We need to show that (i) $\gamma_{0}, \gamma_{1}$ have no pure strategies that are weakly dominated 
or payoff equivalent to a mixed strategy that is not strictly dominated; (ii) $\gamma_{0} \sim_{R A T} \gamma_{1} ;$ (iii) $\gamma_{0} \sim_{C E} \gamma_{1}$; and (iv) $\gamma_{0} \sim_{N E} \gamma_{1}$.

(i) This is shown in von Stengel (2002), p. 1740.

(ii) Since a nondegenerate game has neither redundant nor weakly dominated strategies that are not strictly dominated strategies, and since all games along the path between $\gamma_{0}$ and $\psi\left(\gamma_{1}\right)$ are nondegenerate, the number of stategies surviving iterated strict dominance must be constant along the path and hence $\gamma_{0} \sim{ }_{R A T} \gamma_{1}$.

(iii) To see that two nondegenerate equivalent games are correlated equivalent, we first recall the following well-known lemma, (see for example Fudenberg and Tirole (1991), Ch. 2, p. 57).

Lemma 6 Let $\gamma=(A, B) \in \mathbb{R}^{2 m n}$ be a two person game in normal form. Then $p \in \mathbb{R}^{m n}$ is a correlated equilibrium of $\gamma$ if and only if it is a solution to the linear inequalities:

$$
C p \geq 0,1_{m n} p=1, \text { and } p \geq 0,
$$

where $C$ is the $(m(m-1)+n(n-1)) \times m n$ matrix defined by:

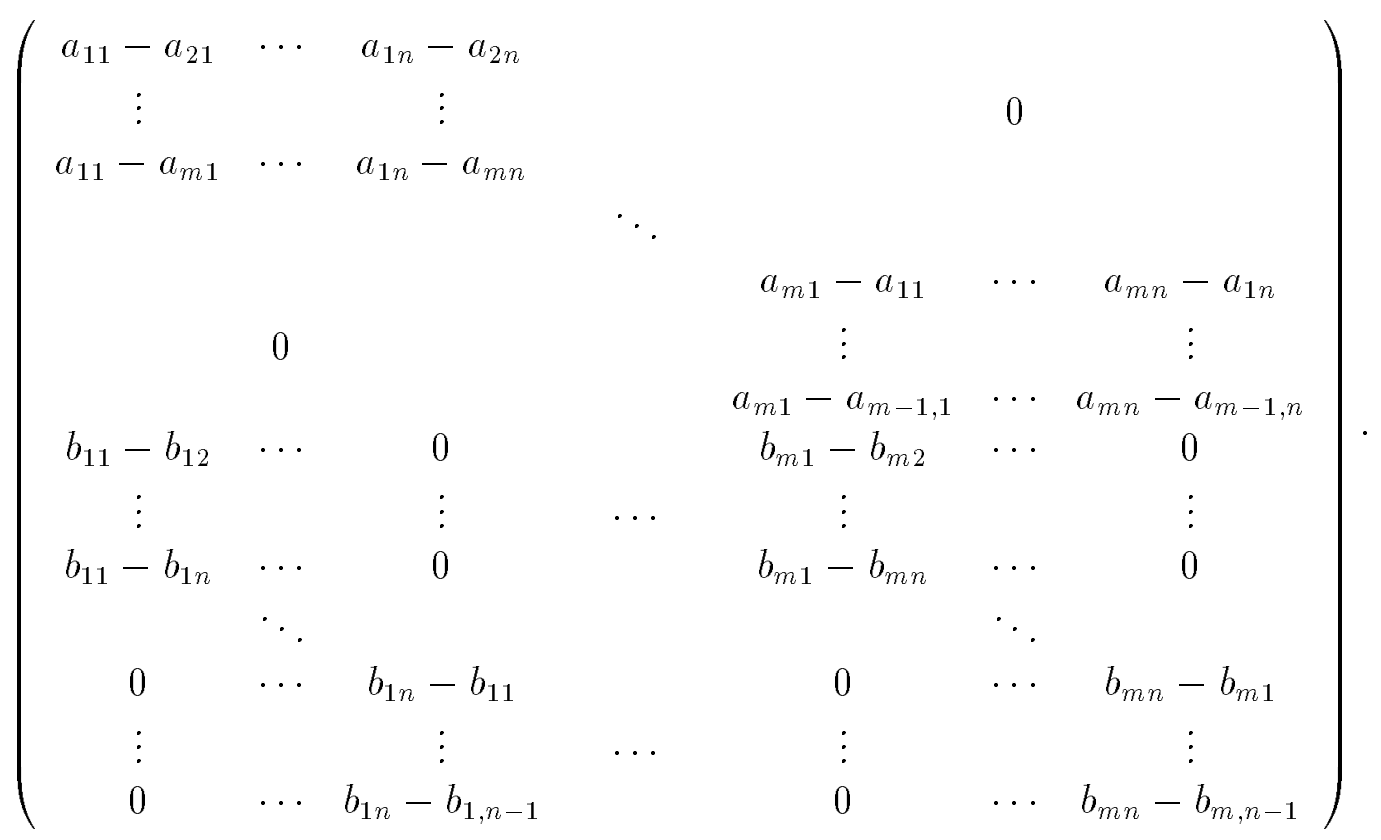

Let $E$ be the matrix consisting of all rows of $\left(C 1_{m n} I_{m n}\right)^{T}$, that hold with equality for all vectors $p \in \mathbb{R}^{m n}$ satisfying (2) at a given game $\gamma^{10}$ Then, as

\footnotetext{
${ }^{10}$ We say a row of $C$ or $I_{m n}$ holds with equality or strict inequality at $\gamma$, if the corresponding inequality of (2) holds with equality or with strict inequality.
} 
a direct application of a well-known result from polyhedral theory, (see, e.g., Nemhauser and Wolsey (1988), Ch. 2, p. 87), it follows that the dimension of the set of correlated equilibria of $\gamma$ is equal to $m n-\operatorname{rank}(E)$. Therefore, it suffices to show that the rank of $E$ is locally constant at $\gamma$. To avoid confusion, we will fix $E$ to consist of all rows holding with equality at the given, nondegenerate game $\gamma$, so that as we change $\gamma$, we keep the set of rows of $E$ fixed while allowing the entries to vary. This means that the only way the dimension of the correlated equilibria can change as $\gamma$ changes is $(i)$ that the rank of $E$ changes or $(i i)$ that there exists a row of $\left(C 1_{m n} I_{m n}\right)^{T}$ not belonging to $E$ at $\gamma$ that holds with equality for all correlated equilibria of a close by game $\gamma^{\prime}$ and moreover is linearly independent from the rows of $E$ at $\gamma^{\prime}$. We show that neither case can arise if $\gamma$ is nondegenrate. First, notice that if a row of $\left(C 1_{m n} I_{m n}\right)^{T}$ does not belong to $E$, then it must be linearly independent from the rows of $E$ at $\gamma$. And if it is linearly independent from $E$, and the span of the rows of $E$ does not increase with small perturbations of $\gamma$, it will continue to be linearly independent from the rows of $E$, and moreover, there will be correlated equilibria of the perturbed games at wich such a row will continue to hold with strict inequality. Therefore, it remains to show that the span of the rows of $E$ cannot be increased by slight perturbations of $\gamma$ when $\gamma$ is nondegenrate.

Suppose that $\gamma$ is nondegenerate. Then, by (i) above, it has neither redundant nor weakly dominated strategies that are not strictly dominated. Since the number of linearly independent rows of $E$ cannot decrease locally, it suffices to show that it cannot increase either. To show this, we make use of the following lemma.

Lemma 7 Let $\gamma=(A, B) \in \mathbb{R}^{2 m n}$ be nondegenerate two person game in normal form and $C$ as above, let $D$ be any submatrix obtained from $\left(C 1_{m n}\right)^{T}$ by deleting all rows corresponding to strictly dominated strategies and possibly more, ${ }^{11}$ then $D$ has full row rank, i.e., $\operatorname{rank}(D)=\min \{m n$, number of rows of $D\}$.

Before proving this lemma, we conclude the proof of the theorem. Notice that any strictly dominated strategy has probability zero at any correlated equilibrium, which implies that any row of $C$ corresponding to a strictly dominated strategy either holds trivially with equality for all correlated equiliria (i.e., because all corresponding entries of the correlated equilibria are zero), or it holds with strict inequality for some correlated equilibrium. (This can be seen by inspection of the matrix $C$ above.) This implies that all the rows of $E$ that correspond to strictly dominated strategies are ones

\footnotetext{
${ }^{11}$ We say a row of $C$ corresponds to a strategy $s_{k}^{i} \in S_{i}$ if it contains $a_{k s}$, for some $s=1, \ldots, n$, when $i=1$ or if it contains $b_{t k}$ for some $t=1, \ldots, m$, when $i=2$.
} 
where all corresponding entries of all correlated equilibria are zero, which in turn implies that the corresponding rows of $I_{m n}$ must also be contained in $E$. In other words the rows of $E$ that correspond to strictly dominated strategies are spanned by corresponding rows of $I_{m n}$. Hence the span of these rows together with the corresponding rows of $I_{m n}$ cannot be increased by varying $\gamma$ locally. It remains to see whether it is possible to increase the span of the remaining rows, i.e., whether it is possible to increase the rank of the matrix $D$ obtained from $E$ after deleting all rows corresponding to strictly dominated strategies as well as the corresponding rows of $I_{m n}$. By the lemma above, such a matrix has full row rank. This implies that it is not possible, by perturbing its entries to increase its rank. As a consequence, perturbing $\gamma$ will not increase the rank of such a submatrix $D$. But then, we have shown that perturbing $\gamma$ will not affect the rank of $E$, and hence the dimension of the set of correlated equilibria must remain locally constant.

(iv) Finally, to see that two nondegenerate equivalent games are Nash equivalent, it follows from van Damme (1991), Ch. 3, pp. 52-55, that at a nondegenerate game all Nash equilibria are locally unique and hence $e_{N E}$ is continuous at such games.

Proof of Lemma 3. It is shown in the text that at most there can be the equivalence classes $\left[\gamma_{1}\right],\left[\gamma_{2}\right],\left[\gamma_{4}\right],\left[\gamma_{5}\right]$. Direct inspection of the payoffs, shows that games in differnt classes cannot be nondegenerate equivalent since ties must occur in the payoffs when going across the different classes.

Proof of Lemma 4. This follows from Kajii and Morris (1997a, b).

Proof of Lemma 7. It suffices to consider the statement of the lemma for the case where $D$ is the full matrix $C$, assuming that the original game has no strictly dominated strategies. For, if a game is nondegenerate, then, the reduced game obtained after iteratedly eliminating all strictly dominated strategies will continue to be nondegenerate. Moreover, it will have neither redundant nor weakly dominated strategies. But then, inspection of the matrix $C$ shows that it must have full row rank since the columns of any set of at least $m n$ rows of $C$ are linearly independent. To see this it may be useful to rewrite the matrix $C$ as the difference of two matrices $C_{1}$ and $C_{2}$ in the obvious way, and recall that regularity of a game with no strictly dominated strategies implies that the submatrices of $A$ and $B^{T}$ appearing in $C_{2}$ will have full row rank (see von Stengel (2002), p. 1740). This implies that any set of at most $m n$ rows of $C$ are linearly independent, and the statement of the lemma then follows. 


\section{References}

[1] Ahn, T.K., Ostrom, E., Schmidt, D., Shupp, R., and J. Walker (2002) "Cooperation in PD Games: Fear, Greed, and History of Play," Public Choice, 106: 137-155.

[2] Bárány, I., J. Lee, and M. Shubik (1992) "Classification of Two-Person Ordinal Bimatrix Games," International Journal of Game Theory, 21: $267-290$.

[3] Battalio, R., L. Samuelson, and J. van Huyck (2001) "Optimization Incentives and Coordination Failure in Laboratory Stag Hunt Games," Econometrica, 69: 749-764.

[4] Berlekamp, E.R., J.H. Conway, and R.K. Guy (2003) Winning Ways for Your Mathematical Plays, I-IV, 2nd ed., A.K. Peters, San Jose, CA.

[5] Blume, L.E. and W.R. Zame (1994) "The Algebraic Geometry of Perfect and Sequential Equilibrium," Econometrica, 62: 783-794.

[6] Cabrales, A., R. Nagel, and R. Armenter (2002) "Equilibrium Selection through Incomplete Information in Coordination Games: An Experimental Study," UPF Working Paper No. 601.

[7] Carlsson, H. and E. van Damme (1993) "Global Games and Equilibrium Selection," Econometrica, 61: 989-1018.

[8] Conway, J.H. (2001) On Numbers and Games, 2nd ed., A.K. Peters, San Jose, CA.

[9] Crawford, V.P. (1997) "Theory and Experiment in the Analysis of Strategic Interaction," in D.M. Kreps and K.F. Wallis (eds.), Advances in Economics and Econometrics: Theory and Applications, I, Seventh World Congress of the Econometric Society, Cambridge University Press, Cambridge.

[10] Erev, I., and A.E. Roth (1998) "Modeling how people play games: Reinforcement Learning in Experimental Games with Unique Mixed Strategy Equilibria," American Economic Review, 88: 848-881.

[11] Friedman, D. (1996) "Equilibrium in Evolutionary Games: Some Experimental Results," Economic Journal, 106: 1-25.

[12] Fudenberg, D. and J. Tirole (1991) Game Theory, MIT Press, Cambrgidge, MA. 
[13] Germano, F. (1998) "On Nash Equivalence Classes of Generic Normal Form Games," CORE Discussion Paper No. 9833.

[14] Gilboa, I., and D. Schmeidler (2001) "Case-Based Decision Theory," Quarterly Journal of Economics, 110: 605-639.

[15] Guy, R.K. (1991) "What is a Game?," Proceedings of Symposia in Applied Mathematics, 43: 1-22.

[16] Harris, R.J. (1972) "An Interval Scale Classification for all $2 \times 2$ Games," Behavioral Science, 17: 371-383.

[17] Harsanyi, J., and R. Selten (1988) A General Theory of Equilibrium Selection in Games, MIT Press, Cambridge, MA.

[18] Holyoak, K.J., and P. Thagard (1995) Mental Leaps: Analogy in Creative Thought, MIT Press, Cambridge, MA.

[19] Jehiel, P. (2002) “Analogy-Based Expectation Equilibrium," Mimeo, CERAS, Paris.

[20] Kajii, A. and S. Morris (1997a) "The Robustness of Equilibria to Incomplete Information," Econometrica, 65: 1283-1309.

[21] Kajii, A. and S. Morris (1997b) "Refinements and Higher Order Beliefs: A Unified Survey," Mimeo, Yale University.

[22] Keiding, H. (1997) "On the Maximal Number of Nash Equilibria in an $n \times n$ Bimatrix Games," Games and Economic Behavior, 21: 148-160.

[23] McKelvey, R.D., and A. McLennan (1997) "The Maximal Number of Regular Totally Mixed Nash Equilibria," Journal of Economic Theory, 72: 411-425.

[24] McLennan, A. (1997) "The Maximal Generic Number of Pure Nash Equilibria," Journal of Economic Theory, 72: 408-410.

[25] Mertens, J.-F. (1987) "Ordinality in Non-Cooperative Games," CORE Discussion Paper No. 8782.

[26] Morris, S., R. Rob, and H.S. Shin (1995) " $p$-Dominance and Belief Potential," Econometrica, 63: 145-157. 
[27] Morris, S., and H.S. Shin (2003) "Global Games: Theory and Applications," in M. Dewatripont, L.P. Hansen, S.J. Turnovsky (eds.), Advances in Economics and Econometrics: Theory and Applications, Eighth World Congress of the Econometric Society, Cambridge University Press, Cambridge.

[28] Morris, S., and T. Ui (2002) "Best Response Equivalence," Mimeo, Yale University and Yokohama National University.

[29] Myerson, R.B. (1991) Game Theory, Harvard University Press, Cambridge, MA.

[30] Nash, J. (1951) “Non-Cooperative Games," Annals of Mathematics, 54: $286-295$.

[31] Nemhauser, G.L. and L.A. Wolsey (1988) Integer and Combinatorial Optimization, Wiley and Sons, New York.

[32] Ochs, J. (1995) "Coordination Problems," in J.H. Kagel and A.E. Roth (eds.), Handbook of Experimental Economics, Princeton University Press, Princeton, NJ.

[33] Pearce, D.G. (1984) "Rationalizable Strategic Behavior and the Problem of Perfection," Econometrica, 52: 1029-1050.

[34] Rapoport, A., and M. Guyer (1966) "A Taxonomy of $2 \times 2$ Games," General Systems, 11: 203-214.

[35] Rapoport, A., M.J. Guyer, and D.G. Gordon (1976) The $2 \times 2$ Game, University of Michigan Press, Ann Arbor.

[36] Roth, A.E. (1995) "Introduction to Experimental Economics," in J.H. Kagel and A.E. Roth (eds.), Handbook of Experimental Economics, Princeton University Press, Princeton, NJ.

[37] Rubinstein, A. (1988) "Similarity and Decision-Making Under Risk," Journal of Economic Theory, 46: 143-153.

[38] Samuelson, L. (2001) "Analogies, Adaptation, and Anomalies," Journal of Economic Theory, 97: 320-366.

[39] Schanuel, S.H., L.K. Simon, and W.R. Zame (1991) "The Algebraic Geometry of Games and the Tracing Procedure," in R. Selten (ed.), Game Equilibrium Models, II: Methods, Morals, and Markets, Springer Verlag, Berlin. 
[40] Straub, P.G. (1995) "Risk Dominance and Coordination Failures in Static Games," The Quarterly Review of Economics and Finance, 35: 339-363.

[41] Van Damme, E. (1991) Stability and Perfection of Nash Equilibria, 2nd ed., Springer Verlag, Berlin.

[42] Van Damme, E. (2002) "Strategic Equilibrium," in R. Aumann and S. Hart (eds.), Handbook of Game Theory, III, Elsevier, Amsterdam.

[43] Van Huyck, J., and R. Battalio (2002) "Prudence, Justice, Benevolence, and Sex:Evidence from Similar Bargaining Games," Journal of Economic Theory, 104: 227-246.

[44] Von Stengel (1999) "New Maximal Numbers of Equilibria in Bimatrix Games," Discrete and Computational Geometry, 21: 557-568.

[45] Von Stengel, B. (2002) "Computing Equilibria for Two-Person Games," in R. Aumann and S. Hart (eds.), Handbook of Game Theory, III, Elsevier, Amsterdam. 